\title{
The role of cell free DNA and liquid biopsies in haematological conditions
}

\author{
Nicholas Bingham, Andrew Spencer \\ Australian Centre for Blood Diseases, Monash University, Victoria 3181, Australia. \\ Correspondence to: Dr. Nicholas Bingham, Australian Centre for Blood Diseases, Monash University, Victoria 3181, Australia. \\ E-mail: nicholas.bingham@monash.edu
}

How to cite this article: Bingham N, Spencer A. The role of cell free DNA and liquid biopsies in haematological conditions. Cancer Drug Resist 2020;3:521-31. http://dx.doi.org/10.20517/cdr.2019.93

Received: 11 Oct 2019 First Decision: 6 Jan 2020 Revised: 21 Jan 2020 Accepted: 18 Feb 2020 Available online: 13 Mar 2020

Science Editor: Godefridus J. Peters Copy Editor: Jing-Wen Zhang Production Editor: Jing Yu

\begin{abstract}
Cell free nucleic acids (CFNAs) are nucleic acids released from cells that circulate within bodily fluids. Recent advances in molecular techniques have led to the ability to interrogate CFNAs in a clinically meaningful way, for example the identification and assessment of foetal CFNAs in maternal blood, allowing minimally invasive testing for foetal genetic abnormalities. The majority of CFNAs arise from haemopoietic cells, making it a particularly rich source of genetic information in haematological conditions. Furthermore, the innate genetic heterogeneity of haematological malignancies, as epitomised by multiple myeloma, lend itself well to "liquid biopsies". This approach promises to provide a more wholistic assessment of whole disease genetics, especially when contrasted against the current gold-standard of single site tissue biopsies. This review briefly summarises the definitions and physiology of CFNAs, both cell free DNA (cfDNA) and extracellular RNA (exRNA), before exploring the literature surrounding the current and future roles of cfDNA in the haematological malignancies and patient care.
\end{abstract}

Keywords: cfDNA, liquid biopsy, haematology, myeloma, lymphoma, leukaemia

\section{INTRODUCTION}

Cell free nucleic acids (CFNAs) were first identified in the blood in $1948^{[1]}$, although many decades passed before molecular techniques with adequate sensitivity to usefully interrogate the CFNAs were developed. Given the relative ease with which most body fluids are accessed, the nucleic acids found in blood and other body fluids are becoming an increasingly attractive source of genetic material for many

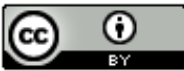

(C) The Author(s) 2020. Open Access This article is licensed under a Creative Commons Attribution 4.0 International License (https://creativecommons.org/licenses/by/4.0/), which permits unrestricted use, sharing, adaptation, distribution and reproduction in any medium or format, for any purpose, even commercially, as long as you give appropriate credit to the original author(s) and the source, provide a link to the Creative Commons license, and indicate if changes were made.

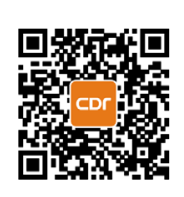


applications. The first cancer-associated mutations identified in CFNAs were in blood taken from patients with myelodysplastic syndrome ${ }^{[2,3]}$ and indeed most cell free DNA (cfDNA) is derived from haemopoietic cells ${ }^{[4,5]}$. Accordingly, the study of CFNAs is tightly interconnected with haematology, and it is in haematological conditions that interrogation of CFNA arguably holds the most promise.

This review briefly summarises the definitions and physiology of CFNAs relevant to liquid biopsies in haematology, with a focus on what is known about cfDNA in haematological conditions and the future potential of liquid biopsies in the clinic.

\section{DEFINITIONS}

The nucleic acids found in the plasma exist as either DNA or RNA. cfDNA describes all of the DNA found in the plasma, while circulating tumour DNA (ctDNA) is the portion of cfDNA that is derived solely from tumour cells. cfDNA is comprised of short fragments of double-stranded $\mathrm{DNA}^{[5]}$, often wrapped around histone proteins (the subunit known as a nucleosome) sometimes with an additional H1 protein (known as a chromatosome). cfDNA is mainly found free in the plasma as these chromatosomes. Historically, it was considered that a small proportion of cfDNA was found within exosomes, the small membrane-bound vesicles released from cells into body fluids ${ }^{[6,7]}$, although more recent data suggest this is not the case ${ }^{[8]}$. This may relate to differences in the methods of exosome isolation, with resultant differences in exosome purity accounting for this observed variation.

RNA can also be found circulating in the blood as extracellular RNA (exRNA); however, the majority of exRNA is found within exosomes ${ }^{[9]}$. The systematic interrogation of exRNA and thus the identification of clinical applications of exRNA in haematology are significantly less developed than cfDNA, as such this review focuses on the role cfDNA in haematological conditions.

\section{PHYSIOLOGY OF CFNA}

CFNAs are released into body fluids from cells dying due to apoptosis or necrosis ${ }^{[5]}$. The release of CFNAs can be spontaneous ${ }^{[10,11]}$ or triggered by chemotherapy- or radiation therapy-induced apoptosis. After cell death, macrophages in the tissue phagocytose cellular debris, but some nucleic acids escape this process and enter the blood stream ${ }^{[12]}$. In addition, it has been recognised that some cells also actively secrete nucleic acids into the body fluids ${ }^{[6,13]}$.

CFNAs are released by tumour cells as well as normal, healthy cells. Moreover, the amount of CFNA found in the blood varies during certain physiological or pathological states. However, it has been long established that the quantity of cfDNA is higher in patients with malignancies ${ }^{[14]}$, averaging $180 \mathrm{ng} / \mathrm{mL}$ compared to $30 \mathrm{ng} / \mathrm{mL}$ in healthy controls ${ }^{[15,16]}$. The ctDNA released from tumour cells comprises a variable proportion of this increase, with a range of $3 \%-93 \%^{[11]}$. Other non-malignant conditions also increase the levels of cfDNA, with elevated levels of cfDNA, when compared to normal individuals, having been identified in a number of disease states, including allogeneic transplantation ${ }^{[17]}$, autoimmune diseases, trauma and after exercise $^{[18,19]}$. In pregnant women, $10 \%-15 \%$ of cfDNA is of foetal origin and this is now routinely used to detect foetal chromosomal abnormalities ${ }^{[20]}$.

cfDNA is released as short fragments of double-stranded DNA, with a typical length of 166-167 base pairs ${ }^{[5,21]}$. Interestingly, ctDNA is usually shorter than cfDNA, with an average length of 132-145 base pairs $^{[21]}$. The typical lengths of ctDNA and cfDNA of 147 and 167 base pairs correlate with the length of DNA associated with a nucleosome and a chromatosome, respectively ${ }^{[22]}$. A total of 147 base pairs represents the length of DNA wrapped around the four histone proteins that comprise a nucleosome, 

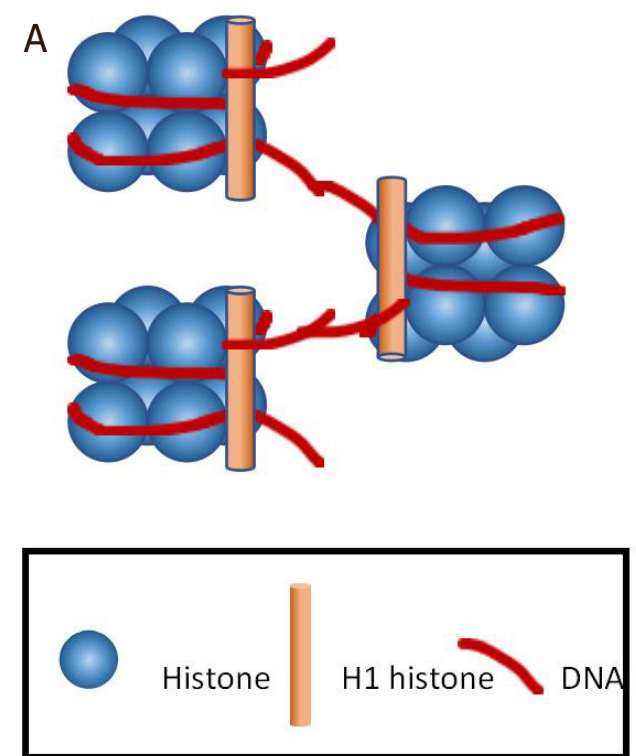

B

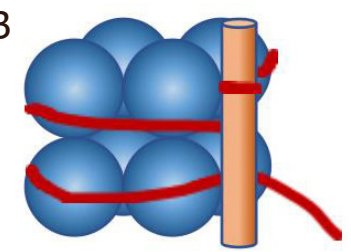

C

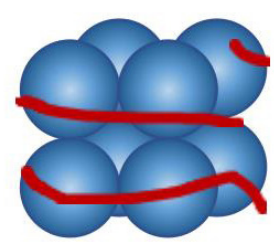

Figure 1. DNA is stored within a cell as chromatin, comprising a single DNA strand wrapped around multiple subunits known as chromatosomes and nucleosomes. A: several subunits are linked by DNA to form chromatin. cfDNA is found as both chromatosomes and nucleosomes; $\mathrm{B}$ : a chromatosome is comprised of a core of eight histone proteins, wrapped in DNA of typically 167 base pairs length, with the linkage section of DNA and the $\mathrm{H} 1$ histone; $\mathrm{C}$ : by contrast, a nucleosome subunit comprises the eight-histone core without the $\mathrm{H} 1$ histone protein and loss of the linkage segment of DNA, making the typical length of DNA in the nucleosome only 147 base pairs in length. cfDNA: cell free DNA

whereas 167 base pairs is the nucleosome DNA with a section of linker DNA attached ${ }^{[5,22]}$ [Figure 1]. Importantly, the association of cfDNA with the nucleosome proteins protects it from cleavage by nucleases ${ }^{[22]}$. Circulating CFNAs are rapidly cleared by the kidneys and liver from the circulation with a half-life measured in minutes to several hours ${ }^{[15]}$, whereas the cfDNA found within exosomes is likely to be protected from this rapid degradation ${ }^{[6,7]}$.

\section{ANALYSING CFDNA IN HAEMATOLOGICAL CONDITIONS}

In mature B-cell non-Hodgkin's lymphomas (NHL) and plasma cell dyscrasias (PCD), malignant clones arise from post-germinal centre B-cells, having undergone rearrangement of the immunoglobulin heavy chain gene as part of the process of somatic hypermutation and immunoglobulin class switching to optimise the affinity of immunoglobulins for specific antigens ${ }^{[23]}$. Theoretically, therefore, all the clonal cells present in either NHL or PCD patients contain identically mutated, patient-specific IgH variable regions.

This clonally rearranged Ig heavy chain DNA was first identified in cfDNA in $1997^{[24]}$, and now, with the development of next-generation sequencing techniques (NGS), it is possible to detect and quantify the IgH gene rearrangements within the cfDNA of both NHL and multiple myeloma (MM) patients ${ }^{[25]}$.

In addition to IgH sequencing, cfDNA can be used to identify disease-specific somatic mutations in haematology patients ${ }^{[26]}$. Once an appropriate driver mutation is identified, subsequent digital PCR can be used to monitor changes in the level of the mutation over time ${ }^{[26]}$ and these changes can be quantitative biomarkers of both disease response and relapse.

An additional utility of cfDNA interrogation is the possibility of examining patterns of DNA methylation. Methylated cfDNA was identified and described in patients with lymphoproliferative disorders in $2003^{[27]}$. In a subsequent study, DLC-1 gene methylation in NHL patients was compared between blood and tumour 
tissue samples and found to be concordant between $\operatorname{sites}^{[28]}$. In this context, methylation patterns can be used to monitor disease progress and may also be of prognostic significance, as discussed below.

\section{Limitations of cfDNA}

There are several inherent limitations in the use of cfDNA in haematological conditions. The use of cfDNA may be confounded in some settings due to the presence of coincident clonal haemopoiesis of indeterminant significance (CHIP) ${ }^{[29]}$. CHIP is a condition with an incidence that increases with age, characterised by a population of clonal haemopoietic cells in the circulation. As most cfDNA is derived from the haemopoietic system ${ }^{[4,5]}$, the presence of CHIP will be associated with the presence of cfDNA derived mutations in the circulation that are separate from an underlying, unrelated malignancy. CHIPassociated mutations are well characterised, and thus may be excluded when cfDNA analysis is being performed for non-haematological malignancies. However, when cfDNA is being used to interrogate a possible haematological condition, CHIP mutations may be indistinguishable from mutations sometimes identified in the condition of interest.

Further to this, many haematological conditions lack tumour-specific mutations with identical mutations found across a range of diverse haematological conditions. Additionally, the clonally rearranged Ig heavy chain requires individually tailored primers to identify the mutation, making the process labour-intensive and expensive and requiring the availability of suitable diagnostic tissue for initial characterisation of the specific IgH mutation ${ }^{[30]}$. Similar to the situation found with coincident CHIP, recurrent somatic mutations can be detected but will not be diagnostic of a specific haematological disease, mandating the requirement for ongoing conventional diagnostic testing.

There are also more general technical limitations for the use of cfDNA that impact the application of cfDNA approaches in haematological conditions and more broadly. Accurate genomic information from cfDNA is critically reliant on the isolation of good quality DNA from samples, requiring standardised and validated protocols for gathering samples and extracting $\mathrm{cfDNA}^{[31]}$. However, there is still a measurable rate of test failure despite these approaches ${ }^{[32]}$. The interrogation of cfDNA can also be affected by the short length of cfDNA fragments, for example in the library preparation step for sequencing reactions ${ }^{[33]}$. Furthermore, the low relative abundance of mutated alleles in cfDNA also impacts the sensitivity of this approach. As cfDNA contains DNA from all tumours throughout the body, and thus all disease subclones, the relative abundance of the individual mutations present is less. It is further diluted amongst the cfDNA from healthy cells. Thus, cfDNA requires highly sensitive next generation techniques with sufficient read depth or digital PCR to target specific mutations that may be present at lower allelic frequencies ${ }^{[18,34]}$.

Technical limitations aside, the major limitations related to the utilisation of cfDNA applications is a lack of randomised control trials that guide its use in the clinic. While undoubtedly providing genomic information and correlating with outcomes, there is at this time no evidence that altering treatment on the basis of cfDNA findings changes patient outcomes when compared to current clinical practice. It is, however, worth noting that in non-haematological conditions cfDNA is in regular clinical use, including foetal karyotyping in pregnancy and in epidermal growth factor receptor mutation testing in lung cancer $^{[2,35]}$. Ongoing clinical trials now frequently include cfDNA analyses to establish clinical utility, but to date data supporting a role for cfDNA in the haematology clinic are preliminary.

\section{cfDNA in MM}

MM is the archetypal haematological disease for studying the utility of cfDNA and liquid biopsies. The marked spatial and temporal genetic heterogeneity of MM is well established, and the gold standard bone marrow biopsy is now recognised as not being genomically representative of the disease as a whole ${ }^{[36-38]}$. cfDNA, representing DNA from the tumour in its entirety, better captures the genetic diversity of MM compared with bone marrow (BM) samples alone ${ }^{[35-38]}$. 
Traditionally, disease monitoring of MM patients involves sequential serum paraprotein estimation, although oligosecretory and non-secretory MM patients require regular bone marrow biopsies or positron emission tomography/computed tomography (PET/CT) scans to monitor disease, which are invasive, costly or involve radiation associated risks. Furthermore, in advanced disease, MM progression does not infrequently manifest as light chain escape, where paraprotein estimations are no longer representative of disease burden, with tumour cells secreting only light chains, or indeed becoming entirely non-secretory ${ }^{[39]}$. Evidently, a non-invasive but representative method of disease burden monitoring that can be used in all MM patients would be highly desirable; cfDNA could potentially fulfil this role. As described below, analysis of cfDNA can provide prognostic as well as genomic information; it can also be used to measure response to treatment, monitor for disease progression and has been correlated with survival.

With regards to prognosis in $\mathrm{MM}$, a higher number of mutations and higher fractional abundance (proportion of mutated versus wild type alleles from a specific locus) of these mutations detected in ctDNA at the start of treatment correlates with worse survival ${ }^{[38]}$. Furthermore, in the same study, response to therapy was predicted by a reduction in the levels of ctDNA at Day 5 , highlighting the promise of ctDNA in the early detection of patients failing therapy ${ }^{[39]}$. The possibility of predicting non-responders and an early change of therapy is an attractive theoretical way to improve patient outcomes, although clinical trials are required to confirm this.

In $\mathrm{MM}$, the end-stages of disease are characterised by the development of extramedullary plasmacytomas, where clonal plasma cell tumours arise outside of the BM niche and are typically refractory to chemotherapy ${ }^{[40]}$. cfDNA is of particular interest in this group of patients, given the difficulty in obtaining biopsies, and that different sites frequently yield different genetic abnormalities ${ }^{[36]}$. Targeted amplicon sequencing of cfDNA using a panel of MM-associated genes has been used in conjunction with multiple biopsies and PET-CT scanning in patients with extramedullary (EM) disease, generating informative quantitative and qualitative data ${ }^{[37]}$. Mutations were identified in cfDNA not present in the BM but found at sites of EM disease, indicating cfDNA may be able to characterise EM disease without a biopsy. Furthermore, changes in cfDNA over time correlated with tumour burden, indicating a possible role in monitoring response to therapy. This study also demonstrated clonal evolution in the cfDNA of a MM patient over time, coinciding with the development of light chain escape and disease progression. This emphasises the power of cfDNA from both diagnostic and monitoring perspectives in advanced MM.

These findings were validated in a separate study that examined the clonally rearranged IgH gene in MM utilising an NGS technique, in contrast to a targeted panel of known recurrent mutations ${ }^{[41]}$. This study found that the clonal IgH rearrangements in cfDNA became undetectable in patients who responded to therapy before a change in the paraprotein, suggesting cfDNA is a more immediate estimation of tumour burden than the traditional methods of monitoring. This approach is limited by the issues with IgH analysis mentioned above but validates the ability of cfDNA to monitor response in MM patients.

Finally, cfDNA analysis in MM can be correlated with survival. In one study of MM patients, paired mutational analysis of both bone marrow DNA and plasma ctDNA in both newly diagnosed and relapsed refractory cases were examined ${ }^{[42]}$. Patients with more than two mutations, or a fractional abundance of a mutation greater than $1 \%$, had significantly shorter overall survival. These findings were validated by another study, where a higher absolute number of mutations, as well as a higher fractional abundance of these mutations, was also associated with worse overall survival ${ }^{[38]}$.

\section{cfDNA in lymphomas}

There is literature surrounding cfDNA in a number of Hodgkin's and NHL, but arguably the best developed area of haematology is in diffuse large B-cell lymphoma (DLBCL). DLBCL is the most common subtype of 
NHL. In the field of DLBCL, cfDNA has been demonstrated to be useful in a number of areas, including diagnosis, prognosis and treatment. For a number of reasons, DLBCL as a disease stands to benefit from the use of cfDNA technology. The current standard for monitoring DLBCL response to therapy is PET/CT scans or other imaging techniques ${ }^{[43]}$, with associated radiation risks and a limit of detection that may miss residual disease. DLBCL diagnosis is based on morphology and the immunophenotype of tumour cells, but there is increasing use of molecular testing, for example to identify cell-of-origin or other targetable mutations that provide useful prognostic information ${ }^{[43]}$. This emerging need for molecular analysis may benefit from increasing use of cfDNA. Studies have used cfDNA in the diagnosis of DLBCL and analysis of the tumour genome, as well as in prognostication and monitoring of patients in remission.

There is promise for cfDNA in the diagnosis in some DLBCL subtypes. Current diagnostic criteria based on morphology and immunophenotype may be problematic in situations where a suitable tissue sample is difficult to obtain but cfDNA identified in body fluids may allow the identification of important diagnostic information. An excellent example is primary central nervous system lymphoma, an aggressive subtype of DLBCL that is difficult to diagnose because of the inherent risks of central nervous system (CNS) tissue biopsies. cfDNA has been identified in the cerebrospinal fluid of patients with CNS tumours ${ }^{[4,45]}$. The identification of lymphoma-associated mutations or clonal $\mathrm{IgH}$ rearrangements within the cerebrospinal fluid may provide a surrogate diagnostic criterion in these cases, although further studies are required to confirm the utility of this approach before it enters clinical use ${ }^{[46]}$.

cfDNA has also been used for the genotyping of DLBCL. In one study, NGS-based techniques were used to correlate the presence of lymphoma-associated genes in the cfDNA and tumour biopsies ${ }^{[34]}$. This study identified mutations in the blood that were the same as the diagnostic tissue biopsy with a concordance rate of $85 \%$. Further studies have used ancer Personalised Profiling by deep sequencing, a technique validated and FDA-approved in non-small cell lung cancer, to interrogate ctDNA in DLBCL ${ }^{[47-49]}$. Rossi et al. ${ }^{[49]}$ demonstrated that mutations with an allele frequency of $>20 \%$ in the primary tumour sample were reliably detectable in the cfDNA compartment.

Importantly, tumour genotyping by cfDNA does not simply mirror the genotype obtained from tumour tissue biopsies; it is complementary. Spatial and temporal heterogeneity of haematological neoplasms is increasingly recognised ${ }^{[50]}$, and cfDNA interrogation provides a representation of the whole-tumour genome rather than that of a single biopsy site. These mutations, identified in cfDNA but not in original tumour tissue biopsies, have been confirmed to be real in a number of confirmatory studies ${ }^{[4,49,51]}$.

Specific mutations, when identified in DLBCL, are known to predict response to treatment. For example, MYD88 mutations may correlate with response to ibrutinib ${ }^{[52]}$. Early studies have shown that current techniques can identify such mutations in $\mathrm{cfDNA}^{\left[{ }_{3}\right]}$. Prospective studies are required before cfDNA is used to guide treatment choices, but the increasing use of personalised medicine in other fields indicates the potential value of such a technique.

In DLBCL, cfDNA can also provide prognostic information. Using IgH sequencing, Kurtz et al. ${ }^{[30]}$ showed that quantification of ctDNA in DLBCL patients correlates closely with metabolic tumour volume. Scherer et al. ${ }^{[48]}$ identified a prognostic correlation using an NGS-based technique, showing ctDNA is a strong predictive factor at diagnosis, with higher levels of ctDNA correlating with inferior progression free survival and a higher risk of disease progression. Interestingly, in the pre-transplant setting, a higher ctDNA level prior to allogeneic stem cell transplant for lymphoma was not prognostic of outcome, but the change in ctDNA levels after transplant correlated with response and predicted relapse ${ }^{[54]}$. Aberrant methylation of cfDNA has also been identified in DLBCL patients and has been correlated with survival ${ }^{[55,56]}$. In one study, the methylation of key lymphoma-associated genes detectable in the cfDNA was assessed, finding that aberrant 
methylation of the DAPK1 gene was associated with worse five-year survival ${ }^{[57]}$. This study was validated in part by a further study that identified global hypomethylation of DNA in both tumour and blood samples of DLBCL patients, showing global hypomethylation was associated with aberrant DAPK1 promoter methylation and shared the same poor prognostic impact ${ }^{[58]}$.

One of the more exciting implications of cfDNA in DLBCL is that it can be used to assess response to therapy and monitor for relapse. In a retrospective study, patients who subsequently progressed were identified; those who had persistent ctDNA in the plasma had the shortest time to progression ${ }^{[59]}$. In patients treated for DLBCL, ctDNA became undetectable in patients who responded to therapy, while it remained detectable in those patients who were resistant, frequently with the emergence of new mutations ${ }^{[49]}$. Response can be predicted by the change in ctDNA levels between Days 0 and 15 of therapy, with a failure to decrease levels at Day 15 predicting a lack of response with high specificity in one study ${ }^{[60]}$. In some lymphomas, interim PET scans are used to escalate or reduce chemotherapy based on response to initial cycles of chemotherapy; this is not yet used in DLBCL. While the above studies of ctDNA show that there is a correlation between interim ctDNA measurement and outcome, there are no studies that show altering treatment based on this result will improve a patient's outcome and further studies are required to confirm this prior to integration into clinical practice.

Other lymphomas with cfDNA data include classic Hodgkin's lymphoma, where detection of specific mutations in plasma correlated with tissue biopsies and may be of prognostic significance but confirmatory studies are pending ${ }^{[6]]}$. Intravascular large B-cell lymphoma, a notoriously difficult diagnosis to make due to its intravascular location, has also been investigated with targeted sequencing and digital PCR of cfDNA, identifying B-cell associated mutations in $88 \%$ of diagnostic samples in one small study ${ }^{[62]}$. There is minimal literature about cfDNA in T-cell lymphomas, likely due to the rarity of these conditions, although one paediatric study found lower proportions of ctDNA in anaplastic large cell lymphoma when compared to $\mathrm{B}$-cell or Hodgkin's lymphomas ${ }^{[63]}$. In angioimmunoblastic T-cell lymphoma, another challenging diagnosis typically requiring multiple biopsies, one study showed that interrogation of cfDNA can detect recurrent mutations and that these mutations became undetectable in patients who responded to therapy ${ }^{[6]]}$.

\section{Myeloid disorders}

There is far less literature about the role of cfDNA in myeloid disorders, such as myeloproliferative disorders, myelodysplastic syndromes (MDS) and acute myeloid leukaemias (AML). In MDS and AML, the ease of access to circulating disease would intuitively suggest a reduced benefit of cfDNA compared to tumour-based DNA assessments. Interestingly, this is not the case. One study examined 24 patients with AML comparing BM and cfDNA samples, finding that cfDNA identified more mutations than found in the $\mathrm{BM}^{[50]}$ with a median of three cfDNA mutations in $83 \%$ of patients that were not detectable in the BM sample. Furthermore, a significant number of patients had potentially actionable mutations found in cfDNA that were not seen in the BM samples. This highlights once again the marked spatial genetic heterogeneity of haematological malignancies, including those with circulating disease.

In AML and MDS patients after allogeneic stem cell transplant, the monitoring of ctDNA can be used to predict relapse and the presence of ctDNA at one month and three months post-transplant correlates with survival ${ }^{[6,66]}$. This may identify patients in whom early interventions, such as weaning of immunosuppression, may reduce relapse rates. Furthermore, ctDNA can be used for chimerism studies to analyse engraftment kinetics ${ }^{[65]}$.

The myelodysplastic syndromes are another area of haematology where cfDNA may prove highly beneficial in filling an unmet need. Current diagnostic criteria rely on morphology and cytogenetic abnormalities; despite the application of strict criteria, the interpretation of morphology remains subjective, and cytogenetic abnormalities are useful but are only found in $50 \%$ of cases ${ }^{[67]}$. Furthermore, genetic 
information is often compromised by hypocellularity of bone marrow samples. Molecular techniques have identified recurrent somatic mutations in MDS, although these are not yet included in diagnostic criteria. cfDNA may therefore represent a useful, easily accessible source of genetic material for recurrent sampling in MDS patients.

In a study of MDS patients on a clinical trial, serial bone marrow and cfDNA samples were analysed; 10/12 patients had driver mutations identified on the BM through next-generation sequencing ${ }^{[68]}$. Digital PCR was performed to validate these results; importantly, the variant allele fractions correlated well between the two sites, and this occurred even when there were marked peripheral blood cytopenias. Importantly, there was no correlation between cfDNA and the white blood cell count in peripheral blood, which suggests the source of cfDNA, while haemopoietic, is the BM rather than peripheral blood cells ${ }^{[68]}$. This study also identified clonal evolution in several patients, including the emergence of mutations associated with transformation to acute leukaemia, and the ctDNA levels changed reflecting tumour burden.

\section{Other haematological conditions}

Another disease in which cfDNA may play a key role in the future is aplastic anaemia (AA). AA is an autoimmune condition where T-cell mediated destruction of haemopoietic stem cells leads to a markedly hypocellular bone marrow and peripheral blood cytopenias; the hypocellularity of the marrow renders traditional techniques to analyse the underlying genetics insensitive ${ }^{[69]}$. In practice, AA is frequently associated with clonal disorders of haemopoiesis, including MDS and paroxysmal nocturnal haemoglobinuria. A study of AA patients identified that mutations were identified more frequently in cfDNA than in BM samples, with $18 \%$ of patients having detectable mutations in cfDNA but not the $\mathrm{BM}^{[69]}$. Thus, cfDNA may be useful in AA patients, but also displays a proof of concept in patients with hypocellular bone marrow that historically makes genetic assessment challenging.

\section{CONCLUSION AND FUTURE DIRECTIONS}

Interrogation of cfDNA via liquid biopsy holds promise in a range of haematological conditions. cfDNA provides genetic information at diagnosis that can be missed by other biopsy techniques, providing complementary information to current gold standard diagnostic techniques. Changes in cfDNA over time correlate with survival and response to therapy, and changes in cfDNA can predict disease progression.

These findings open the door for cfDNA to guide therapeutic decisions in the clinic, for example through the identification of targetable lesions at diagnosis as well as by changing therapies on the basis of early prediction of poor response. There is less literature surrounding cfDNA in myeloid conditions; however, the ability of cfDNA analysis to identify genetic abnormalities in many myeloid conditions is undisputable.

The next step for cfDNA in haematology is incorporation into clinical trials to better characterise the clinical utility of these findings. Some questions to answer are challenging: What should be done when a patient is in a minimal residual disease negative remission by traditional minimal residual disease assessments, but is positive by cfDNA? What is the optimal therapy for a patient who has a targetable mutation in the cfDNA that is not identified on the traditional biopsy? There is indisputable promise in cfDNA for the haematology patient, but such questions need to be addressed in clinical trials before cfDNA can be confidently applied for haematology patients in the clinic.

\section{DECLARATIONS}

\section{Authors' contributions}

Wrote and reviewed the manuscript: Bingham N, Spencer A 


\section{Availability of data and materials}

Not applicable.

\section{Financial support and sponsorship}

None.

\section{Conflicts of interest}

All authors declared that there are no conflicts of interest.

\section{Ethical approval and consent to participate}

Not applicable.

\section{Consent for publication}

Not applicable.

\section{Copyright}

(c) The Author(s) 2020.

\section{REFERENCES}

1. Mandel P, Metais P. Les acides nucléiques du plasma sanguin chez l’homme. C R Seances Soc Biol Fil 1948;142:241-3.

2. Vasioukhin V, Anker P, Maurice P, Lyautey J, Lederrey C, et al. Point mutations of the N-ras gene in the blood plasma DNA of patients with myelodysplastic syndrome or acute myelogenous leukaemia. Br J Haematol 1994;86:774-9.

3. Sorenson GD, Pribish DM, Valone FH, Memoli VA, Bzik DJ, et al. Soluble normal and mutated DNA sequences from single-copy genes in human blood. Cancer Epidemiol Biomarkers Prev 1994;3:67-71.

4. Lui YYN, Chik KW, Chiu RWK, Ho CY, Lam CWK, et al. Predominant hematopoietic origin of cell-free DNA in plasma and serum after sex-mismatched bone marrow transplantation. Clin Chem 2002;48;421-7.

5. Snyder MW, Kircher M, Hill AJ, Daza RM, Shendure J. Cell-free DNA comprises an in vivo nucleosome footprint that informs its tissues-of-origin. Cell 2016;164:57-68.

6. Peters DL, Pretorius PJ. Origin, translocation and destination of extracellular occurring DNA--a new paradigm in genetic behaviour. Clin Chim Acta 2011;412:806-11.

7. Kahlert C, Melo SA, Protopopov A, Tang J, Seth S, et al. Identification of double-stranded genomic DNA spanning all chromosomes with mutated KRAS and p53 DNA in the serum exosomes of patients with pancreatic cancer. J Biol Chem 2014;289:3869-75.

8. Jeppesen DK, Fenix AM, Franklin JL, Higginbotham JN, Zhang Q, et al. Reassessment of exosome composition. Cell 2019;177:428-45. e18.

9. Valadi H, Ekström K, Bossios A, Sjöstrand M, Lee JJ, et al. Exosome-mediated transfer of mRNAs and microRNAs is a novel mechanism of genetic exchange between cells. Nat Cell Biol 2007;9:654-9.

10. Fournié GJ, Courtin JP, Laval F, Chalé JJ, Pourrat JP, et al. Plasma DNA as a marker of cancerous cell death. Investigations in patients suffering from lung cancer and in nude mice bearing human tumours. Cancer Lett 1995;91:221-7.

11. Jahr S, Hentze H, Englisch S, Hardt D, Fackelmayer FO, et al. DNA fragments in the blood plasma of cancer patients: quantitations and evidence for their origin from apoptotic and necrotic cells. Cancer Res 2001;61:1659-65.

12. Choi JJ, Reich CF, Pisetsky DS. The role of macrophages in the in vitro generation of extracellular DNA from apoptotic and necrotic cells. Immunology 2005;115:55-62.

13. Stroun M, Lyautey J, Lederrey C, Olson-Sand A, Anker P. About the possible origin and mechanism of circulating DNA apoptosis and active DNA release. Clin Chim Acta 2001;313:139-42.

14. Leon SA, Shapiro B, Sklaroff DM, Yaros MJ. Free DNA in the serum of cancer patients and the effect of therapy. Cancer Res 1977;37:646-50.

15. Fleischhacker M, Schmidt B. Circulating nucleic acids (CNAs) and cancer--a survey. Biochim Biophys Acta 2007;1775:181-232.

16. Sunami E, Vu AT, Nguyen SL, Giuliano AE, Hoon DSB. Quantification of LINE1 in circulating DNA as a molecular biomarker of breast cancer. Ann N Y Acad Sci 2008;1137;171-4.

17. Snyder TM, Khush KK, Valantine HA, Quake SR. Universal noninvasive detection of solid organ transplant rejection. Proc Natl Acad Sci U S A 2011;108:6229-34.

18. Wan JCM, Massie C, Garcia-Corbacho J, Mouliere F, Brenton JD, et al. Liquid biopsies come of age: towards implementation of circulating tumour DNA. Nat Rev Cancer 2017;17:223-38.

19. De Vlaminck I, Martin L, Kertesz M, Patel K, Kowarsky M, et al. Noninvasive monitoring of infection and rejection after lung transplantation. Proc Natl Acad Sci U S A 2015;112:13336-41. 
20. Chiu RWK, Chan KCA, Gao Y, Lau VYM, Zheng W, et al. Noninvasive prenatal diagnosis of fetal chromosomal aneuploidy by massively parallel genomic sequencing of DNA in maternal plasma. Proc Natl Acad Sci U S A 2008;105:20458-63.

21. Underhill HR, Kitzman JO, Hellwig S, Welker NC, Daza R, et al. Fragment length of circulating tumor DNA. PLoS Genet 2016;12:e1006162.

22. Fan HC, Blumenfeld YJ, Chitkara U, Hudgins L, Quake SR. Noninvasive diagnosis of fetal aneuploidy by shotgun sequencing DNA from maternal blood. Proc Natl Acad Sci U S A 2008;105:16266-71.

23. Cooper MD. The early history of B cells. Nat Rev Immunol 2015;15:191-7.

24. Frickhofen N, Müller E, Sandherr M, Binder T, Bangerter M, et al. Rearranged Ig heavy chain DNA is detectable in cell-free blood samples of patients with b-cell neoplasia. Blood 1997;90:4953-60.

25. He J, Wu J, Jiao Y, Wagner-Johnston N, Ambinder RF, et al. IgH gene rearrangements as plasma biomarkers in Non- Hodgkin's lymphoma patients. Oncotarget 2011;2:178-85.

26. Wu FT, Lu L, Xu W, Li JY. Circulating tumor DNA: clinical roles in diffuse large B cell lymphoma. Ann Hematol 2018;98:255-69.

27. Deligezer U, Yaman F, Erten N, Dalay N. Frequent copresence of methylated DNA and fragmented nucleosomal DNA in plasma of lymphoma patients. Clin Chim Acta 2003;335:89-94.

28. Shi H, Guo J, Duff DJ, Rahmatpanah F, Chitima-Matsiga R, et al. Discovery of novel epigenetic markers in non-Hodgkin's lymphoma. Carcinogenesis 2007;28:60-70.

29. Kammesheidt A, Tonozzi TR, Lim SW, Braunstein GD. Mutation detection using plasma circulating tumor DNA (ctDNA) in a cohort of asymptomatic adults at increased risk for cancer. Int J Mol Epidemiol Genet 2018;9:1-12.

30. Kurtz DM, Green MR, Bratman SV, Scherer F, Liu CL, et al. Noninvasive monitoring of diffuse large B-cell lymphoma by immunoglobulin high-throughput sequencing. Blood 2015;125:3679-87.

31. Mithraprabhu S, Spencer A. Analysis of circulating tumor DNA. Methods Mol Biol 2018;1792:129-45.

32. Kubaczkova V, Vrabel D, Sedlarikova L, Besse L, Sevcikova S. Cell-free DNA - Minimally invasive marker of hematological malignancies. Eur J Haematol 2017;99:291-9.

33. Perdigones N, Murtaza M. Capturing tumor heterogeneity and clonal evolution in solid cancers using circulating tumor DNA analysis. Pharmacol Ther 2017;174:22-6.

34. Bohers E, Viailly PJ, Dubois S, Bertrand P, Maingonnat C, et al. Somatic mutations of cell-free circulating DNA detected by nextgeneration sequencing reflect the genetic changes in both germinal center B-cell-like and activated B-cell-like diffuse large B-cell lymphomas at the time of diagnosis. Haematologica 2015;100:e280-4.

35. Wu YL, Sequist LV, Hu CP, Feng J, Lu S, et al. EGFR mutation detection in circulating cell-free DNA of lung adenocarcinoma patients: analysis of LUX-Lung 3 and 6. Br J Cancer 2017;116:175-85.

36. Rasche L, Chavan SS, Stephens OW, Patel PH, Tytarenko R, et al. Spatial genomic heterogeneity in multiple myeloma revealed by multiregion sequencing. Nat Commun 2017;8:268.

37. Mithraprabhu S, Sirdesai S, Chen M, Khong T, Spencer A. Circulating tumour DNA analysis for tumour genome characterisation and monitoring disease burden in extramedullary multiple myeloma. Int J Mol Sci 2018;19.

38. Mithraprabhu S, Morley R, Khong T, Kalff A, Bergin K, et al. Monitoring tumour burden and therapeutic response through analysis of circulating tumour DNA and extracellular RNA in multiple myeloma patients. Leukemia 2019;33:2022-33.

39. Dawson MA, Patil S, Spencer A. Extramedullary relapse of multiple myeloma associated with a shift in secretion from intact immunoglobulin to light chains. Haematologica 2007;92:143-4.

40. Usmani SZ, Heuck C, Mitchell A, Szymonifka J, Nair B, et al. Extramedullary disease portends poor prognosis in multiple myeloma and is over-represented in high-risk disease even in the era of novel agents. Haematologica 2012;97:1761-7.

41. Oberle A, Brandt A, Voigtlaender M, Thiele B, Radloff J, et al. Monitoring multiple myeloma by next-generation sequencing of V(D)J rearrangements from circulating myeloma cells and cell-free myeloma DNA. Haematologica 2017;102:1105-11.

42. Mithraprabhu S, Hocking J, Ramachandran M, Choi K, Klarica D, et al. DNA-repair gene mutations are highly prevalent in circulating tumour DNA from multiple myeloma patients. Cancers (Basel) 2019;11.

43. Tilly H, Gomes da Silva M, Vitolo U, Jack A, Meignan M, et al. Diffuse large B-cell lymphoma (DLBCL): ESMO Clinical Practice Guidelines for diagnosis, treatment and follow-up. Ann Oncol 2015;26:v116-25.

44. De Mattos-Arruda L, Mayor R, Ng CKY, Weigelt B, Martínez-Ricarte F, et al. Cerebrospinal fluid-derived circulating tumour DNA better represents the genomic alterations of brain tumours than plasma. Nat Commun 2015;6:8839.

45. Wang Y, Springer S, Zhang M, McMahon KW, Kinde I, et al. Detection of tumor-derived DNA in cerebrospinal fluid of patients with primary tumors of the brain and spinal cord. Proc Natl Acad Sci U S A 2015;112:9704-9.

46. Fontanilles M, Marguet F, Bohers É, Viailly PJ, Dubois S, et al. Non-invasive detection of somatic mutations using next-generation sequencing in primary central nervous system lymphoma. Oncotarget 2017;8:48157-68.

47. Newman AM, Bratman SV, To J, Wynne JF, Eclov NC, et al. An ultrasensitive method for quantitating circulating tumor DNA with broad patient coverage. Nat Med 2014;20:548-54.

48. Scherer F, Kurtz DM, Newman AM, Stehr H, Craig AF, et al. Distinct biological subtypes and patterns of genome evolution in lymphoma revealed by circulating tumor DNA. Sci Transl Med 2016;8:ra155.

49. Rossi D, Diop F, Spaccarotella E, Monti S, Zanni M, et al. Diffuse large B-cell lymphoma genotyping on the liquid biopsy. Blood 2017;129:1947-57.

50. Assi RE, Albitar M, Ma W, Patel K, Takahashi K, et al. Comparison of somatic mutations profiles from next-generation sequencing (NGS) of cell-free DNA (cfDNA) versus bone marrow (BM) in acute myeloid leukemia (AML). J Clin Oncol 2018;36:7051. 
51. Murtaza M, Dawson SJ, Tsui DW, Gale D, Forshew T, et al. Non-invasive analysis of acquired resistance to cancer therapy by sequencing of plasma DNA. Nature 2013;497:108-12.

52. Wilson WH, Young RM, Schmitz R, Yang Y, Pittaluga S, et al. Targeting B cell receptor signaling with ibrutinib in diffuse large B cell lymphoma. Nat Med 2015;21:922-6.

53. Camus V, Sarafan-Vasseur N, Bohers E, Dubois S, Mareschal S, et al. Digital PCR for quantification of recurrent and potentially actionable somatic mutations in circulating free DNA from patients with diffuse large B-cell lymphoma. Leuk Lymphoma 2016;57:2171-9.

54. Herrera AF, Kim HT, Kong KA, Faham M, Sun H, et al. Next-generation sequencing-based detection of circulating tumour DNA after allogeneic stem cell transplantation for lymphoma. Br J Haematol 2016;175:841-50.

55. Barault L, Amatu A, Bleeker FE, Moutinho C, Falcomatà C, et al. Digital PCR quantification of MGMT methylation refines prediction of clinical benefit from alkylating agents in glioblastoma and metastatic colorectal cancer. Ann Oncol 2015;26:1994-9.

56. Bedin C, Enzo MV, Del Bianco P, Pucciarelli S, Nitti D, et al. Diagnostic and prognostic role of cell-free DNA testing for colorectal cancer patients. Int J Cancer 2017;140:1888-98.

57. Kristensen LS, Hansen JW, Kristensen SS, Tholstrup D, Harsløf LB, et al. Aberrant methylation of cell-free circulating DNA in plasma predicts poor outcome in diffuse large B cell lymphoma. Clin Epigenetics 2016;8:95.

58. Wedge E, Hansen JW, Garde C, Asmar F, Tholstrup D, et al. Global hypomethylation is an independent prognostic factor in diffuse large B cell lymphoma. Am J Hematol 2017;92:689-94.

59. Roschewski M, Dunleavy K, Pittaluga S, Moorhead M, Pepin F, et al. Circulating tumour DNA and CT monitoring in patients with untreated diffuse large B-cell lymphoma: a correlative biomarker study. Lancet Oncol 2015;16:541-9.

60. Assouline SE, Nielsen TH, Yu S, Alcaide M, Chong L, et al. Phase 2 study of panobinostat with or without rituximab in relapsed diffuse large B-cell lymphoma. Blood 2016;128:185-94.

61. Camus V, Stamatoullas A, Mareschal S, Viailly PJ, Sarafan-Vasseur N, et al. Detection and prognostic value of recurrent exportin 1 mutations in tumor and cell-free circulating DNA of patients with classical Hodgkin lymphoma. Haematologica 2016;101:1094-101.

62. Suehara Y, Sakata-Yanagimoto M, Hattori K, Nanmoku T, Itoh T, et al. Liquid biopsy for the identification of intravascular large B-cell lymphoma. Haematologica 2018;103:e241-e4.

63. Mussolin L, Burnelli R, Pillon M, Carraro E, Farruggia P, et al. Plasma cell-free DNA in paediatric lymphomas. J Cancer 2013;4:323-9.

64. Sakata-Yanagimoto M, Nakamoto-Matsubara R, Komori D, Nguyen TB, Hattori K, et al. Detection of the circulating tumor DNAs in angioimmunoblastic T- cell lymphoma. Ann Hematol 2017;96:1471-75.

65. Aljurf M, Abalkhail H, Alseraihy A, Mohamed SY, Ayas M, et al. Chimerism analysis of cell-free DNA in patients treated with hematopoietic stem cell transplantation may predict early relapse in patients with hematologic malignancies. Biotechnol Res Int 2016;8589270.

66. Nakamura S, Yokoyama K, Shimizu E, Yusa N, Kondoh K,et al. Personalized circulating tumor DNA predicts relapse after allogeneic hematopoietic stem cell transplantation in acute myeloid leukemia and myelodysplastic syndrome. Blood 2017;133:2682-95.

67. Swerdlow SH, Campo E, Harris NL, Jaffe ES. WHO classification of tumours of haematopoietic and lymphoid tissues. (IARC Who Classification of Tum, 2017).

68. Yeh P, Dickinson M, Ftouni S, Hunter T, Sinha D, et al. Molecular disease monitoring using circulating tumor DNA in myelodysplastic syndromes. Blood 2017;129:1685-90.

69. Albitar A, Townsley D, Ma W, De Dios I, Funari V, et al. Prevalence of somatic mutations in patients with aplastic anemia using peripheral blood cfDNA as compared with BM. Leukemia 2018;32:227-29. 